\title{
SIGNIFICADO DEL CUIDADO HUMANIZADO EN EGRESADAS DE LA FACULTAD DE ENFERMERÍA*
}

\author{
Yolanda Muñoz Hernández ${ }^{* *}$, Rosa Coral Ibarra**, Dayana Moreno Prieto***, Diana Pinilla Pinto ${ }^{* * *}$, \\ Yaneth Suárez Rodríguez***
}

\section{Resumen}

EI propósito es conocer el significado de cuidado humanizado en egresadas de la facultad de enfermería de la Fundación Universitaria de Ciencias de la Salud, para que a través de sus directivos, docentes y estudiantes se unifique para conceptualizarlo y asumirlo. Se realizó un estudio fenomenológico dentro de un diseño cualitativo mediante la aplicación de una guía de observación estructurada y una entrevista a profundidad, a cinco egresadas de la Facultad de Enfermería que laboran en el área clínica del Hospital de San José. Las entrevistas fueron grabadas, luego transcritas y clasificadas en categorías de análisis mediante unidades de significación. Pudo concluirse que el cuidado humanizado significa una atención integral teniendo muy en cuenta la parte humana del paciente, afirman que debe brindarse independiente de que la persona esté sana o enferma y además consideran que es sentir sin involucrarse con el enfermo, permitir la expresión de sentimientos de la persona a quien se cuida y respetar su entorno familiar y personal.

Palabras clave: cuidado humanizado, enfermería.

\section{MEANING OF HUMANIZED CARE IN THE LIGHT OF GRADUATES OF THE SCHOOL OF NURSING*}

\section{Abstract}

The purpose of this paper is to understand what humanized care means to graduates of the school of nursing of the Health Sciences University Foundation, in order to unify, conceptualize and assume this concept through managerial personnel, faculty and students. A qualitative phenomenological study was carried out using a structured observation guide and an in-depth interview applied to five female graduates of the nursing school who work at the clinical area of the San José Hospital. Interviews were recorded, and then transcribed and categorized by units of meaning. It could be concluded that humanized care is to deliver comprehensive care placing special emphasis on the human aspects of individuals. They state it should be offered to all regardless if they are healthy or ill and they also consider it is to feel for the patient without getting involved allowing the individual to express his/her feelings showing respect for his/her family and personal settings.

Key words: humanized care, nursing.

Fecha recibido: enero 5 de 2009 - Fecha aceptado: junio 24 de 2009

* Fundación Universitaria de Ciencias de la Salud. Bogotá DC. Colombia.

** Docentes Facultad de Enfermería, Fundación Universitaria de Ciencias de la Salud. Bogotá D.C. Colombia.
*** Estudiantes VII semestre, Facultad de Enfermería, Fundación Universitaria de Ciencias de la Salud. Bogotá DC. Colombia. 


\section{Introducción}

Teniendo en cuenta que el cuidado a través de la historia ha tenido diferentes variaciones en cuanto a su conceptualización, interpretación y aplicación en la práctica, es prioritario conocer si se ha interiorizado en los profesionales de enfermería para brindarlo de forma integral y humanizada. Cabe destacar que cada acción, llámese procedimiento, manejo de protocolo o de equipos de alta tecnología, utilización de habilidades, destrezas y aplicación de conocimientos científicos, se lleva a cabo en el ejercicio profesional sin desconocer que se está trabajando con personas que merecen afecto, amor, dedicación, comprensión y muchas otras características que implican brindar cuidado humanizado.

Cuando se habla así se pretende profundizar en la concepción de persona, promoviendo su calidad de vida, protegiendo sus derechos, contribuyendo a la satisfacción de sus necesidades sin olvidar los aspectos psicológicos, sociales, espirituales y de su entorno.

El oficio de los enfermeros como cuidadores de la salud se ha desplazado por la urgencia de asistir la enfermedad y asumir las funciones preventivista y promotora de salud, convirtiéndose en una actividad reparadora que obedece más a las demandas de las tareas de curar de la medicina que a las necesidades de mantener y fortalecer la salud. Por otra parte, en esta institucionalización del oficio de cuidar, los enfermeros deben actuar bajo órdenes médicas y su quehacer debe ser guiado sólo por las teorías científicas y la técnica médica, perdiendo así la capacidad y el interés de articular las habilidades y los conocimientos técnicos, con la sabiduría y la ternura. Situación que ha creado en el enfermero un grado de dependencia muy alto de la teoría y la práctica médica.

La acción de cuidar la salud y la vida de los seres humanos se desarrolla a lo largo del ciclo vital de los individuos, es ejercida por cada uno en sí mismo, en el cuidado del ser objeto de su amor, en la ejecución del servicio para aquel que solicita atención; es una acción que puede recaer en el ámbito de la vida cotidiana, en la realización de las acciones de promoción y prevención, las curativas y rehabilitadoras, en el mantenimiento de la vida como en el acompañamiento del desenlace y extinción de la misma.
Cuidar es ante todo un acto de vida, significa movilizar las capacidades del ser humano en su cotidianidad, en el proceso de vida-muerte, en estado de salud o enfermedad, teniendo en cuenta sus costumbres, con el objeto de lograr el desarrollo de su capacidad de vivir o de compensar y suplir la alteración de sus funciones biosociales. Implica acercarse al otro con una actitud ética de apertura, de sensibilidad y de responsabilidad ante las experiencias propias y de los otros y así orientar la aplicación de sus conocimientos y habilidades.

El proceso de cuidar las experiencias de salud de las personas, familias o grupos, además de requerir una dinámica de transacciones humano-humano, requiere de una serie de elementos complejos que el enfermero posee y que abarcan conocimientos bien diferenciados, dedicación y valores humanos, porque en el acto de cuidar el enfermero está inmerso en una relación personal y moral con el sujeto cuidado.

La fenomenología es a la vez una filosofía y un método, y en ambos casos puede orientar la investigación en enfermería para contestar preguntas relacionadas con los fenómenos que son experimentados como parte del cuidado de la salud humana, tal y como él existe, como algo propio de seres corporales actuantes que sienten, piensan, se relacionan con otros y pueden crecer en su capacidad de cuidar. Su meta es describir la experiencia humana tal y como ella es vivida por la gente, dando así un valor prioritario al mundo de nosotros y de los otros.

La fenomenología busca ser un retrato exacto de lo que se experimenta, reconoce la vivencia de estar en el mundo de manera particular y no sus hechos interiores o exteriores. No busca comprobar veracidad sino que la asumen, no pretende sólo explicar ni juzgar, refiere la experiencia que se vive. Sin duda el abordaje fenomenológico es una buena manera de dirigir investigaciones en enfermería, siendo una alternativa rigurosa que se contrapone a la tradición de la investigación positivista y presupone que el investigador conoce el pensamiento fenomenológico antes de comenzar a practicarlo.

Para efectos de la investigación se utilizó una guía de tres preguntas abiertas basadas en las categorías de análisis, las cuales fueron: significado de cuidado, significado de cuidado humanizado y experiencia en la práctica de enfermería. 


\section{Materiales y métodos}

Para la realización de esta investigación se trabajó una metodología de tipo cualitativo fenomenológico, el tipo de muestreo seleccionado por conveniencia base de criterios, para el cual se seleccionaron cinco enfermeras egresadas de la Fundación Universitaria de Ciencias de la Salud (FUCS) que laboran en los diferentes servicios del área clínica del Hospital de San José y manifestaron el deseo voluntario de colaborar en la investigación. El tipo de estudio es fenomenológico, el cual permite comprender la naturaleza del ser humano, la experiencia vivida a través de "la ventana del lenguaje" y pretende dar cuenta de la esencia del significado de una experiencia.

\section{Resultados}

De acuerdo con las entrevistas realizadas a las cinco enfermeras egresadas de la Fundación Universitaria de Ciencias de la Salud que laboran en el Hospital de San José, son relevantes los siguientes aspectos. Teniendo en cuenta el significado expresado por ellas se encontró que para una cuidado es "prestar un servicio", el término evoca la idea de "hacer, de acción" encaminado a la atención "básica y asistencial" lo que se relaciona con las necesidades humanas las que afirman que los individuos tienen sus características propias, pero hay ciertas necesidades comunes a todas las personas como las fisiológicas y psicológicas, afirmando que las primeras deben cubrirse antes que las de seguridad.

En su mayoría, las entrevistadas conciben el cuidado como "una atención integral" lo cual se fundamenta con el concepto filosófico de holismo, en el que se considera al individuo como un ser completo no como un conjunto de partes y compromete no sólo lo físico sino las más altas manifestaciones del espíritu humano, en donde todos los organismos vivos se ven como totalidades interaccionantes y unificadas, más que la simple suma de sus partes; el trastorno de una afecta a todo el ser. Implica la aceptación de su estilo de vida, actitudes y circunstancias vitales, lo que puede ser difícil, sobre todo, cuando estas o su entorno son lesivos.

Una de las enfermeras además de expresar que el cuidado debe ser "una atención integral", afirma que debe brindarse "independiente de que esté sano o enfermo", concepto que se relaciona en forma directa con el comentario expresado en el código de ética de enfermería, en el que el acto de cuidado se desarrolla dentro de una relación privilegiada enfermero-paciente (familia, grupo) que recibe asistencia. Es privilegiada por la continuidad, la confianza e intimidad que lo caracteriza y que le permite al enfermero conocer las necesidades de la persona, tanto aquellas derivadas del estado de salud o enfermedad, como otras situaciones de angustia y preocupación que alteran su bienestar.

Con respecto a la categoría relacionada con el significado de cuidado humanizado, una de ellas lo considera lo mismo que el término cuidado por sí solo, pero agrega que se tiene muy en cuenta la "parte humana del paciente", lo cual es compatible con el enfoque integral de la atención de salud en enfermería, exige comprender y atender todas las interrelaciones significativas de los procesos y elementos internos de la persona humana como un ser valioso en sí mismo y para ser cuidado, respetado, comprendido y asistido; en general, una mirada filosófica de la persona como un ente funcional e integral.

Otra de las enfermeras afirma que "cuidar puede hacerlo cualquier persona, pero de una forma excelente sólo el enfermero", lo cual se fundamenta dentro de la perspectiva del cuidado como un "rasgo humano innato", que es una forma del ser, parte de la naturaleza y existencia humana. Todos los seres humanos tienen un potencial o capacidad de cuidar, pero no es uniforme esta capacidad ni la forma de hacerlo; cuando la enfermera entrevistada se refiere a la excelencia del cuidado, éste se relaciona con el arte de la enfermería, que incluye un cuidado perfecto, estudiado, con una secuencia de pasos congruentes como lo muestran los manuales de enseñanza. El enfermero se prepara para cuidar a través de conocimientos y experiencias educativas, es decir, no lo hace sólo basado en habilidad innata.

Para otra enfermera cuidado humanizado es "sentir sin involucrarme con el paciente", expresión apoyada en el concepto de empatía que exige introducirse en el mundo del paciente, pero no de una forma que anule la distancia ni sea una disolución del yo personal en el ajeno, o a la inversa del yo ajeno en lo personal. Por otra parte, cuando el enfermero afirma "el día que uno deje de sentir 
tristeza por ciertas situaciones, deje de llorar y que le deje de doler que su paciente se está muriendo y usted no puede hacer nada, ese día, debe dejar de ser enfermero". Dicha concepción se relaciona con el término simpatía que se refiere al hecho de compartir el dolor del otro, que desde el punto de vista etimológico significa padecer con..., una disposición que llevaría a confirmar o agravar el sentimiento de impotencia vivido por ambos.

Por último, con respecto al cuidado humanizado, otra de las enfermeras lo entiende como "no enfocarse sólo a la enfermedad, sino en la persona que está a su cuidado", considerándola "un ser inteligente y pensante que posee razón y reflexión", es un ser único, integrado con el medio ambiente, como campo de energía irreductible, sensible y capaz de participar de manera productiva en el cambio. La filosofía en enfermería reconoce que todo hombre es persona, es un ser integral, totalitario, único e irrepetible, que involucrado en un contexto particular piensa, siente y actúa de acuerdo con un conjunto propio de valores, creencias, prácticas, características y experiencias a lo largo de la vida, que lo hace mucho más de lo que a simple vista es observable.

\section{Discusión}

La esencia, el deber ser de la enfermería, es el cuidado, "preocupación por el ser humano", es conocer a la persona, identificar sus pensamientos, sentimientos, dudas, expectativas, dolores, molestias; es ayudar al crecimiento y maduración del individuo. Cuidado humanizado es la expresión dedicada, atenta, incondicional del enfermero al entrar en contacto con el paciente. Es ayudar a que recobre su independencia, su autonomía, es la preocupación y el esmero para lograr un estado de máximo bienestar físico, psicológico, social y espiritual, lo cual se logra a través de la comunicación terapéutica, la comprensión, la aceptación, la tolerancia, la escucha activa, el establecimiento de una relación de ayuda donde los pensamientos y sentimientos se reflejan de manera coherente con las acciones que se realizan.

El cuidado humanizado se apoya en el conocimiento científico, la capacidad técnica y la relación terapéuti- ca que el enfermero establece con el paciente. Es acoger al otro de una manera cálida sin dejar de ser yo mismo, despojándome de todos los factores externos que en algún momento puedan afectar la atención comprometida y de óptima calidad, que implica el cuidado humanizado.

\section{Conclusiones}

- Cuidado significa atención integral al paciente, tener en cuenta al individuo como persona, proporcionando un completo bienestar físico, social y psicológico encaminado a satisfacer las necesidades humanas.

- Cuidado humanizado es enfocarse no sólo en la enfermedad sino en la persona que está a mi cuidado y permitir que mis sentimientos hacia los demás se vean reflejados en la atención a mis pacientes. Es lo mejor que podemos dar, un cuidado ideal, excelente, que sólo el enfermero puede hacer.

\section{Lecturas recomendadas}

1. Colombia. Congreso. Ley 266 de 1996 (enero 25) por la cual se reglamenta la profesión de enfermería en Colombia y se dictan otras disposiciones (Diario Oficial 42710 del 5 de febrero de 1996).

2. Asociación Colombiana de Facultades de Enfermería. VII Coloquio Panamericano de Investigación en Enfermería: memorias de las ponencias centrales y páneles, Bogotá, 9-12 oct., 2000. Bogotá D.C.: La Asociación; 2000. p. 27.

3. Asociación Colombiana de Facultades de Enfermería. Operacionalización del cuidado en la formación del profesional de Enfermería en: Memorias $43^{\circ}$ Consejo de Directoras y $36^{\circ}$ Asamblea General. Bogotá D.C.: La Asociación; 2000. p. 22.

4. Bermejo José. Humanizar la salud. Madrid: Artes Gráficas Humanas; Madrid España, 1997. p. 67.

5. Duque S. Dimensión del cuidado de Enfermería a los colectivos: un espacio para la promoción de la salud y la producción de conocimiento. Invest. educ. Enferm. 1999;17(1):75-85.

6. Durán de V. MM. El cuidado como núcleo de la Enfermería. Universidad Nacional de Colombia. Santafé de Bogotá D.C. 1994. p.3.

7. Universidad Nacional de Colombia. Facultad de Enfermería. Grupo de Cuidado. Cuidado y práctica de Enfermería. Bogotá D.C.: Unibiblos.; 2000

8. Universidad Nacional de Colombia. Facultad de Enfermería. Grupo de Cuidado. Dimensiones del Cuidado.Bogotá D.C.: Unibiblos.; 1997 
9. Montes de V. M. Un modelo fundamentado en el amor para la asistencia y el cuidado de las adolescentes embarazadas. Cali: XYZ; 1999. p. 59.

10. Morrison M. Fundamentos de enfermería en salud mental. Madrid: Harcourt Brace; 1999

11. Prieto de R. Gloria. Humanización del Cuidado de Enfermería en Etica y Enfermería. Boletin Latinoamericano de Ética No. 4 Comité de Ética ACOFAEN Bogotá D.C. 2000, año 4 No. 4 p. 7.
12. Universidad Nacional de Colombia. Facultad de Enfermería. Grupo de Cuidado. Elementos de reflexión para el arte y el oficio de cuidar la salud. Santafé de Bogotá D.C.: Unibiblos; 1999.

13. Universidad Nacional de Colombia. Facultad de Enfermería. Grupo de Cuidado. Reflexiones acerca del concepto de cuidar. Santafé de Bogotá D.C.: Unibiblos; [s.f.]

14. Watson Jean. Nursing: human science and human care. A theory of nursing. Canadá: National League for Nursing; 1994. p.33-34. 